\title{
Die Fontan-Konversion - oder das Schicksal, die Erste zu sein
}

\author{
Kerstin Wustmann ${ }^{\mathrm{a}}$, Alexander Kadner ${ }^{\mathrm{b}}$, Jean-Pierre Pfammatterc, Etienne Delacrétaza, Thierry Carrel ${ }^{b}$, \\ Markus Schwerzmann ${ }^{\text {a }}$ \\ a Universitätsklinik für Kardiologie, Herzchirurgie, Inselspital, Bern \\ b Universitätsklinik für Herzchirurgie, Inselspital, Bern \\ c Universitätsklinik für Kinderkardiologie, Inselspital, Bern
}

\section{Summary}

We report the case of the first patient with tricuspid atresia in whom an "old-fashioned" Fontan operation with an atriopulmonary anastomosis was performed in our hospital 18 years ago. Complications of the former "classical" Fontan circulation can include progressive right atrial dilatation leading to tachyarrhythmias, flow energy loss, obstruction of pulmonary veins, right atrial thrombi and the formation of pulmonary arteriovenous fistulas. In order to palliate our patient from recurrent atrial tachyarrhythmias and increasing haemodynamic disturbances, the previous "old-fashioned" Fontan circulation was surgically revised and converted according to a "modern" type of extracardiac Fontan circulation with additional anti-arrhythmic procedures. The post-operative course was uneventful. After recovery from surgery, the patient's quality of life improved. She is free from atrial tachycardias and shows an increased exercise capacity. We will briefly discuss the complications of the classical Fontan circulation and the possible risks of the Fontan conversion. After having been the first Fontan patient in our center, our patient was again the first to require a major revision of its circulation.

\section{Einleitung}

Die Fontan-Zirkulation ist eine Palliationseingriff komplexer angeborener Herzfehler, bei denen der subpulmonale Ventrikel zu klein oder ineffizient ist, um eine ausreichende Lungenperfusion zu gewährleisten, und eine biventrikuläre Korrektur nicht möglich ist. Die Fontan-Zirkulation beinhaltet das direkte Fortleiten des systemvenösen Rückflusses in die Pulmonalarterien unter Verzicht eines subpulmonalen Ventrikels. Dadurch wird eine Trennung des venösen vom systemischen Blut und somit eine Wiederherstellung

Die Autoren erklären, dass sie keine Interessenkonflikte im Zusammenhang mit diesem Beitrag haben eines Parallelkreislaufes ermöglicht. Auch bei Patienten mit einem hypoplastischen Systemventrikel (z.B. hypoplastic left heart syndrome) wird heutzutage eine Fon-
tan-Zirkulation über mehrere Schritte (Norwood procedure) angelegt, wobei dann der verbleibende (ehemals subpulmonale) Ventrikel die Funktion als Systemventrikel übernimmt. Trotz aller Erfolge in der Kindheit bleibt die Fontan-Zirkulation im späteren Leben eine nicht perfekte Lösung für ein komplexes Problem. Der hier berichtete Fall zeigt, wie die erste Fontan-Patientin am Inselspital Bern erneut die erste Patientin ist, die sich einem weiteren komplexen Eingriff 18 Jahre später zu unterziehen hat.

\section{Fallbeschreibung}

Die Patientin wurde 1984 mit einer Trikuspidalatresie und einem grossen Ventrikelseptumdefekt, einer Transposition der grossen Arterien und einer subvalvulären Pulmonalstenose geboren. In den ersten Lebensjahren bestand eine «balancierte» Physiologie. Die Pulmonalstenose verhinderte die Entwicklung einer pulmonalen Hypertonie. 1992 wurde bei ihr als erste Patientin am Inselspital eine Fontan-Zirkulation angelegt, in der das rechte Vorhofohr mit dem Truncus pulmonalis verbunden wurde (atriopulmonale Anastomose; Abb. 1B). Während der nachfolgenden Jugendzeit war sie körperlich gut belastbar und beschwerdefrei. In den letzten drei Jahren beklagte sie zunehmend rezidivierende, mehrmals täglich auftretende, symptomatische Tachykardien von mehreren Minuten Dauer, die sie im Alltag einschränkten. Als weiteres bestand eine progrediente Leistungsintoleranz, die sich in der Spiroergometrie mit einer Maximalbelastung von 90 Watt und $\mathrm{VO}_{2} \max$ von $21,3 \mathrm{ml} / \mathrm{min} / \mathrm{kg}$ ( $58 \%$ des Solls) ausdrückte. Klinisch bestand eine leichte Hypoxämie mit einem Biox von 90\%. Echokardiographisch zeigten sich eine normale Funktion des linken Ventrikels und

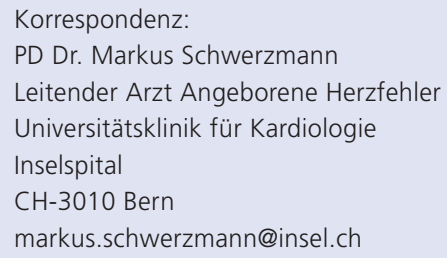




\section{Abbildung 1}

Schematische Darstellung der verschiedenen Typen von möglichen Fontan-Zirkulationen.

A Originale Fontan-Operation mit oder ohne klappentragende Anastomosen des rechten Vorhofes mit der linken Pulmonalarterie sowie der Vena cava superior mit der rechten Pulmonalarterie, unter Ligatur der Pulmonalisbifurkation.

B Atrio-pulmonale Anastomose nach Kreutzer mit Anastomose des rechten Vorhofohres mit der rechten Pulmonalarterie.

C Atrioventrikuläre Anastomose nach Björk bei Trikuspidalatresie.

D Intrakardialer lateraler Tunnel mit Anastomose der Vena cava inferior zur rechten Pulmonalarterie.

E Extrakardiale totale cavo-pulmonale Anastomose mittels Conduit (TCPC) und Fenestrierung.

F TCPC ohne Fenestrierung.

SVC = Vena cava superior; IVC = Vena cava inferior; RPA = rechte Pulmonalarterie; $\mathrm{LPA}=$ linke Pulmonalarterie; $\mathrm{MPA}=$ Truncus pulmonalis.

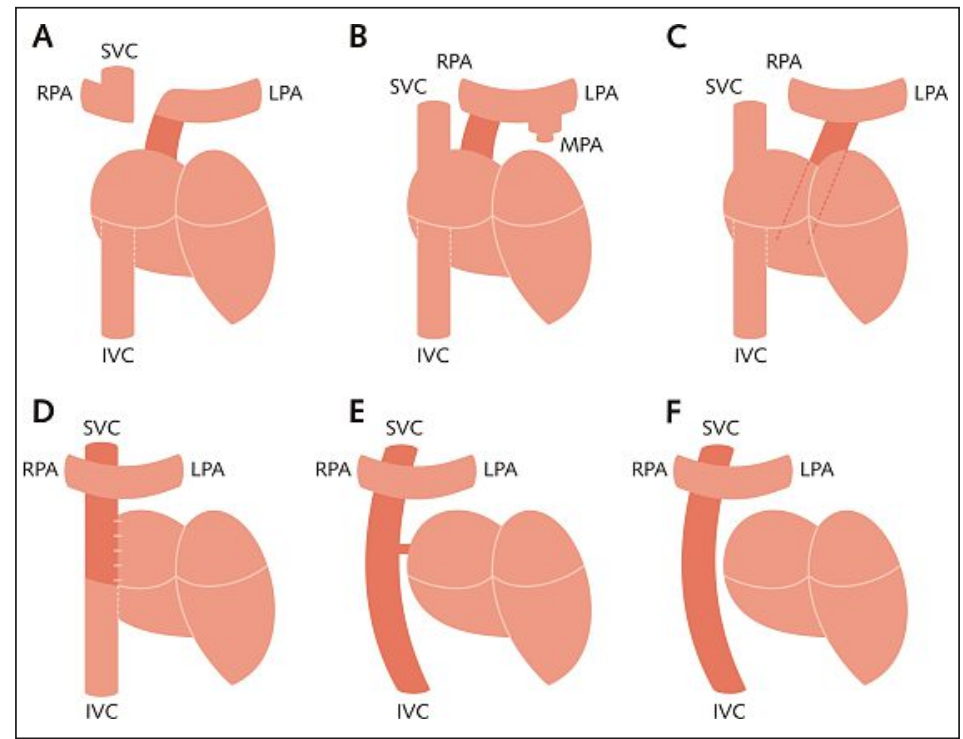

\section{Abbildung 2}

Präoperative transthorakale Echokardiographie in der apikalen «4-Kammer»-Ansicht mit Darstellung des massiv dilatierten rechten Vorhofes (RA) und Vorwölbung des atrialen Septums. Sichtbar sind auch die Gewebebrücke anstelle der Trikuspidalklappe sowie der residuelle «rechte Ventrikel» (RV).

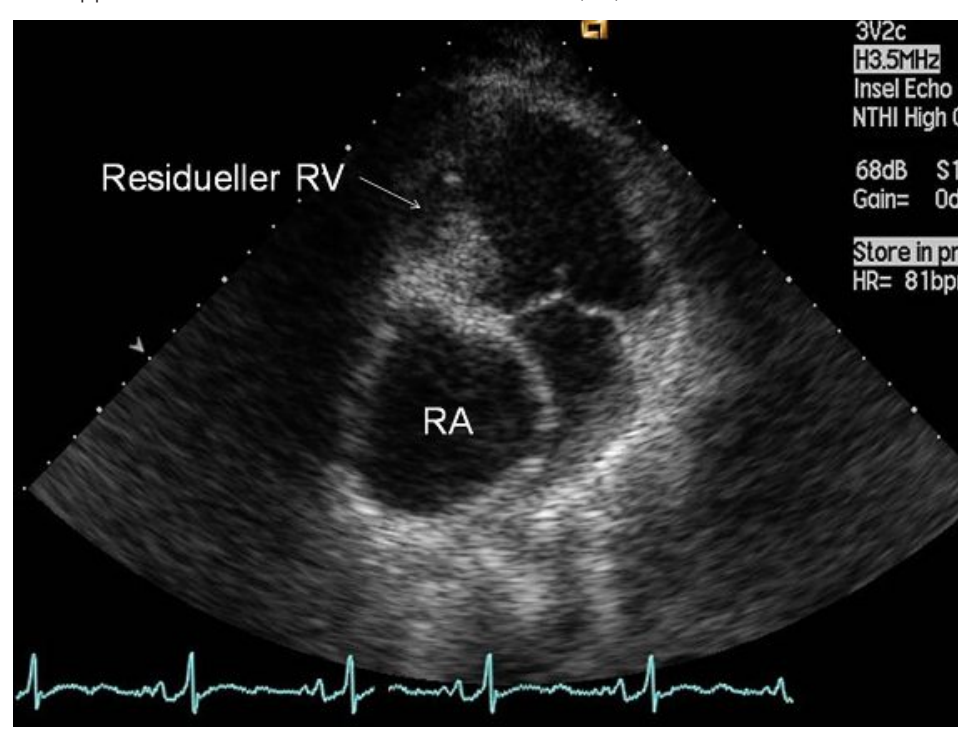

der Klappen sowie ein eindrücklich dilatierter rechter Vorhof (Abb. 2). Durch die Vorhofsdilatation kam es zu einer retrokardialen Kompression der rechtsseitigen Lungenvenen (Abb. 3).

Die invasive Abklärung ergab keine behebbaren lokalen Obstruktionen innerhalb der Fontan-Zirkulation, ebenso konnten aorto-pulmonale, pulmonale arterio-venöse und veno-venöse Shunts ausgeschlossen werden. Der mittlere rechtsatriale Druck betrug 12 $\mathrm{mm} \mathrm{Hg}$, der mittlere pulmonale Druck $11 \mathrm{~mm} \mathrm{Hg}$ und der mittlere pulmonale Wedge-Druck $5 \mathrm{~mm} \mathrm{Hg}$. In der elektrophysiologischen Untersuchung konnten inzisionelle rechtsatriale Reentry-Tachykardien induziert werden, vor allem lateral zwischen Vena cava superior (VCS) und Vena cava inferior (VCI).

In einem interdisziplinären Kolloquium wurden die möglichen Behandlungsoptionen diskutiert. Aufgrund der im Alltag vermehrt eingeschränkten Leistungsfähigkeit und der symptomatischen inzisionellen supraventrikulären Arrhythmien fassten wir mit der Patientin den Entschluss, nicht nur die Arrhythmien anzugehen, sondern gleichzeitig die Fontan-Zirkulation chirurgisch zu verbessern und einen günstigeren Langzeitverlauf und eine bessere Lebensqualität der Patientin zu sichern.

Nach Resternotomie und ausgedehnter Adhäsiolyse, insbesondere des massiv dilatierten rechten Vorhofes, wurde die Patientin mittels arteriell-aortaler und venöser-bicavaler Kannulation an die Herz-Lungen-Maschine angeschlossen. Das rechte Atrium wurde eröffnet und die Anastomose zur Pulmonalarterie identifiziert. Nach ausgedehnter Resektion der antero-lateralen Vorhofswand bis auf ca. 1/4 der ursprünglichen Grösse wurde das intraatriale Septum reseziert. Dies ermöglichte eine ungestörte Drainage des Sinus coronarius in den linken Vorhof. Nach Durchführung einer rechtsatrialen Kryoablation wurde die VCI am Eintritt zum Atrium abgesetzt und der Vorhof verschlossen. Für die Herstellung der VCI-Pulmonalis-Kontinuität wurde eine 19-mm-ringverstärkte PTFE-Gefässprothese gewählt, welche End-zu-End mit der VCI und End-zu-Seit am inferioren Aspekt der zentralen Pulmonalarterie am Ort der vorherigen atriopulmonalen Verbindung anastomosiert wurde. Aufgrund der angestrebten Anastomose mit dem Truncus pulmonalis und der Umgehung des rechten Atriums liess sich eine leichte Bogenform des Goretex-Conduits nicht vermeiden. Die Vena cava superior wurde End-zu-Seit mit der rechten Pulmonalarterie anastomosiert. Abschliessend wurde ein epikardiales Zwei-Kammer-Schrittmachersystem mit Antitachykardie-Funktion implantiert (Abb. 4). Unmittelbar postoperativ wurde die Patientin mit Cordarone aufgesättigt und mit DDD-Modus mit einer Frequenz von 120/min stimuliert. Es wurde eine frühzeitige Extubation angestrebt.

Eine Rechtsherzkatheteruntersuchung aufgrund prolongierter Diarrhoe eine Woche postoperativ zeigte 
Abbildung 3

Präoperatives Kontrastmittel-CT mit Darstellung des stark dilatierten rechten Atriums (RA) und einer konsekutiven partiellen Kompression der rechten Pulmonalvenen.

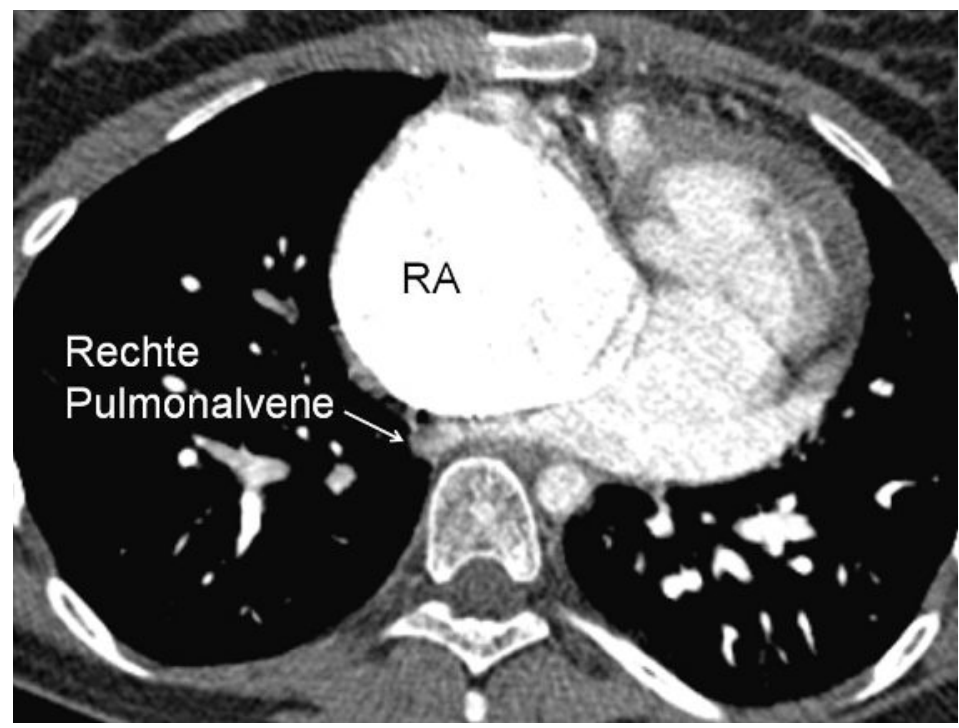

\section{Abbildung 4}

Intraoperativer Situs nach «Fontan-Konversion»: Status nach Implantation eines 19-mm-PTFE-ringverstärkten extrakardialen Conduits (TCPC), Vorhofreduktionsplastie und Implantation von linksatrialen und systemventrikulären epikardialen Vorhofelektroden.

$\mathrm{TCPC}=$ totale cavo-pulmonale Konnektion; $\mathrm{Ao}=$ Aorta; SME = epikardiale Schrittmacherelektroden; SV = Systemventrikel.

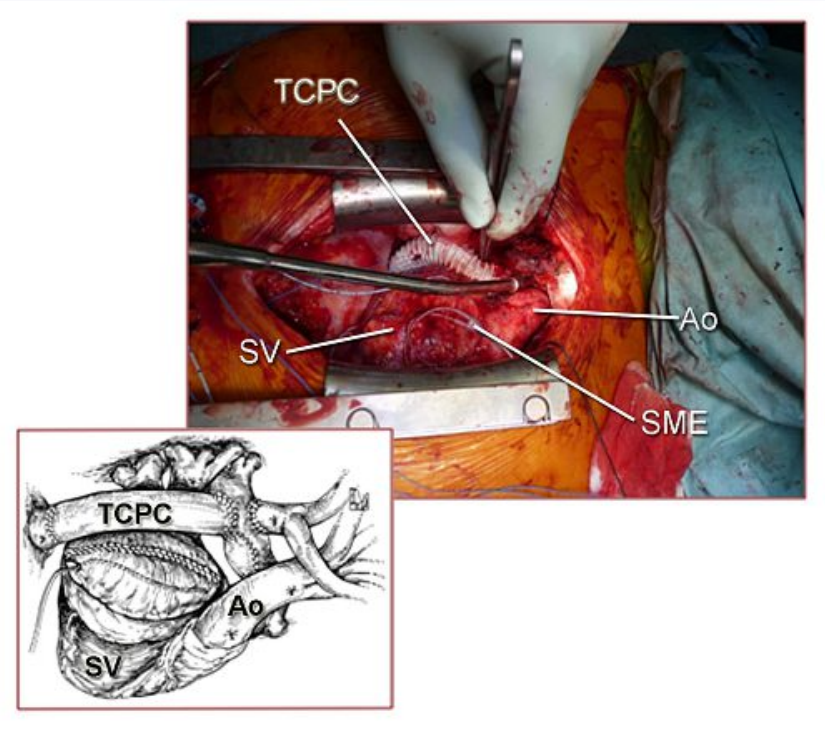

eine gute funktionierende «neue» extrakardiale Fontan-Zirkulation (Abb. 5). In der Schrittmacherabfrage ergaben sich im weiteren Verlauf keine Hinweise für weitere Tachyarrhythmien. Bereits in der vierten postoperativen Woche leistete unsere Patientin in der Eintrittsuntersuchung für die ambulante kardiale Rehabilitation 10 METs (100\% des Solls). Cordarone konnte in der Zwischenzeit abgesetzt werden. Die weiteren Schrittmacherkontrollen zeigten, dass der Grundrhythmus nun ein Sinusrhythmus war, die Patientin aber während der Ruhephasen atrial gepaced wurde (AAIR 70-145/min).

\section{Diskussion}

Die Fontan-Operation wurde ursprünglich von Fontan und Baudet im Jahre 1971 für Patienten mit Trikuspidalatresie eingeführt [1]. Der "typische» Fontan-Patient weist eine Trikuspidalatresie auf und hat einen hypoplastischen rechten Ventrikel, der über einen Ventrikelseptumdefekt mit dem linken Ventrikel in Kommunikation steht. Die Hypoplasie des rechten Ventrikels verunmöglicht eine biventrikuläre Korrektur. Heutzutage wird eine Fontan-Zirkulation auch bei $\mathrm{Pa}$ tienten mit anderen komplexen Herzfehlern und effektiv singulärem Ventrikel, bei denen ebenfalls eine biventrikuläre Korrektur nicht möglich ist, angelegt. Wichtige Voraussetzungen für die Anlage einer FontanZirkulation sind ein tiefer pulmonaler Widerstand, keine relevante systolische Dysfunktion des Ventrikels und keine bedeutende Insuffizienz der AV-Klappe(n) [2].

Seit der ersten Publikation von Fontan und Baudet im Jahre 1971 haben sich die chirurgischen Möglichkeiten, eine Fontan-Zirkulation anzulegen, entscheidend weiterentwickelt (Abb. 1). Wurden bei den ersten Fontan-Patienten noch eine unidirektionale obere cavo-pulmonale Anastomose («klassische GlennAnastomose») sowie klappentragende Anastomosen zwischen der VCI und der atrio-pulmonalen Konnektion durchgeführt (Abb. 1A), setzte sich eine technisch einfachere und weniger komplikationsträchtige Variante nach Kreutzer (wie bei unserer Patientin) für die folgenden Jahre durch. Hierbei wurde lediglich der rechte Vorhof mit dem Truncus pulmonalis anastomosiert (Abb. 1B). Nach weiteren Modifikationsschritten wird heutzutage der rechte Vorhof umgangen und das Blut der VCI durch einen extrakardialen Conduit direkt in die Pulmonalarterie geleitet (Abb. 1E, 1F). Die «Fontan-Zirkulation» wird dabei stufenweise erreicht. Falls nötig wird beim Neugeborenen mit komplexem Herzfehler als Erstes eine pulmonale Hyperperfusion durch Bändelung der Pulmonalarterien oder eine pulmonale Hypoperfusion durch Anlage einer aorto-pulmonalen Verbindung (z.B. modifizierter Blalock-Taussig-Shunt) korrigiert. Als nächster Schritt wird im Alter von 3-6 Monaten zur Vorbereitung der FontanZirkulation bidirektional eine Anastomose zwischen VCS und rechter Pulmonalarterie angelegt. Als letzter Schritt erfolgt im Alter zwischen 2 und 3 Jahren die Implantation eines extrakardialen Conduits zur Konnektion der VCI und der Pulmonalarterie mit der resultierenden kompletten Trennung des venösen und systemischen Kreislaufes. 


\begin{abstract}
Abbildung 5
Angiographie vor (links) und nach Fontan-Revision (rechts). Vor der Revisionsoperation wird das Blut von der Vena cava inferior und den Lebervenen über den riesigen RA in die pulmonale Zirkulation geleitet. Nach der Fontan-Revision zeigt sich der Konfluens der Vena cava inferior mit den Lebervenen in den extrakardialen Goretex-Conduit sowie dessen Anastomose zum Truncus pulmonalis unmittelbar proximal der Bifurkation. Durch die separate Mündung der Vena cava superior in die rechte Pulmonalarterie zeigt sich hier weniger Kontrastmittel (konkurrenzierender Fluss). Die epikardialen linksatrialen und linksventrikulären Elektroden des Schrittmachers sind ebenfalls erkennbar.
\end{abstract}

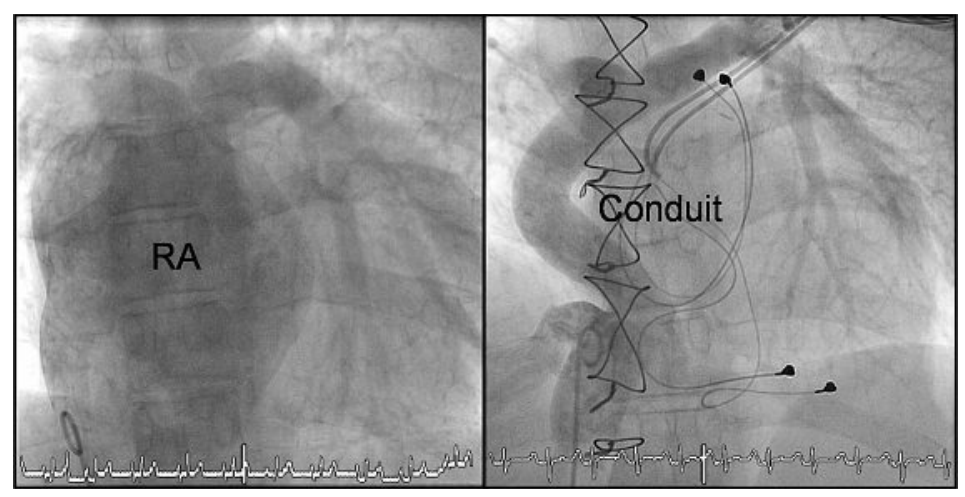

Die vergangenen dreissig Jahre haben gezeigt, dass die Mehrheit der Fontan-Patienten in der Kinderund Jugendzeit beschwerdearm sind. Im späteren Leben sind die physiologischen Konsequenzen des Verzichts auf einen subpulmonalen Ventrikel unausweichlich. Der chronisch erhöhte systemvenöse Druck fordert Tribut, und insbesondere bei Patienten mit atriopulmonaler Anastomose führt die rechtsatriale Dilatation zu atrialen Tachyarrhythmien, ungünstigen Strömungsverhältnissen und Thrombusbildung und kann auch in einer Kompression der Lungenvenen enden. Bei bis zu 10\% der Fälle können sich lymphatische Komplikationen als "Protein-loosing enteropathy» [3] oder «plastische Bronchitis» manifestieren, welche mit einer Mortalitätsrate von bis zu 40\% einhergehen. Intrapulmonale arterio-venöse oder veno-venöse Fisteln sind weitere mögliche Komplikationen [4, 5] und treten v.a. bei Patienten mit klassischem Glenn-Shunt auf. Die supraventrikulären Tachykardien (häufig atriales Makro-Reentry) sprechen typischerweise schlecht auf eine medikamentöse Therapie an. Antitachykardie-Pacing und Ablation sind die am meisten erfolgversprechenden Therapieansätze. Hinzu kommt nicht selten eine systolische und diastolische Dysfunktion des Systemventrikels, bei der aufgrund der speziellen Hämodynamik eine traditionelle Herzinsuffizienztherapie keinen grossen klinischen Benefit zeigt [6].

In dieser Situation liegt der Ausweg in sorgfältig ausgewählten Fällen in einem aggressiven Behandlungsplan, bei dem die Hämodynamik konsequent optimiert und Arrhythmien abladiert werden. Das Stenting von Stenosen innerhalb der Fontan-Zirkulation, der Verschluss von Fisteln oder die Umwandlung einer atriopulmonalen Fontan-Zirkulation in eine extrakardiale totale cavo-pulmonale Konnektion sind Möglichkeiten, die Hämodynamik zu optimieren, ohne auf einen Herzersatz zurückzugreifen. Die Mortalität einer Fontan-Konversion wird in der Literatur in grossen Serien mit 6-20\% angegeben [7]. Perioperative Komplikationen können sich als Blutungen aufgrund der ausgedehnten Adhäsionen, dialysepflichtiges Nierenversagen, kardiales Pumpversagen und Indikation für Herztransplantation, "Protein-loosing enteropathy", persistierende Pleuraergüsse und Mediastinitis manifestieren. In einer grossen Serie mit 111 Patienten aus Chicago, einem der erfahrensten Zentren für FontanKonversionen mit antiarrhythmischer Chirurgie, wurde das transplantationsfreie Überleben drei Jahre postoperativ mit 88\% angegeben. Prädiktive Faktoren für Tod oder Herztransplantation sind mittelschwere bis schwere AV-Klappeninsuffizienz, präoperative «Protein-loosing enteropathy», lange Bypass-Zeit (>239 Minuten) und ein morphologisch rechter Systemventrikel. Atriale Tachykardierezidive traten bei 13,5\% der Überlebenden auf [7].

Unbestritten bleibt die Fontan-Zirkulation eine nicht perfekte Lösung eines komplexen Problems. Obwohl die in unserem Fall beschriebene Patientin mit ihren optimalen pulmonalen Druckverhältnissen, kompetenten Klappen und gut erhaltener Ventrikelfunktion eine Niedrig-Risiko-Patientin für eine Fontan-Zirkulation darstellte, benötigte sie eine chirurgische Korrektur für Spätkomplikationen der altmodischen Fontan-Variante. Diese Patienten bedürfen obligat lebenslange engmaschige kardiologische Verlaufskontrollen in Zentren mit speziell ausgebildeten Kardiologen für angeborene Herzfehler, in enger Zusammenarbeit mit in diesem Gebiet erfahrenen Herzchirurgen, Elektrophysiologen, interventionellen Kardiologen und Herztransplantationsteam.

\section{Literatur}

1 Fontan F, Baudet E. Surgical repair of tricuspid atresia. Thorax. 1971;26(3):240-8.

2 Giroud JM, Jacobs JP. Fontan's operation: evolution from a procedure to a process. Cardiol Young. 2006;16(Suppl 1):67-71.

3 Ostrow AM, Freeze H, Rychik J. Protein-losing enteropathy after fontan operation: investigations into possible pathophysiologic mechanisms. Ann Thorac Surg. 2006;82(2):695-700.

4 Magee AG, McCrindle BW, Mawson J, Benson LN, Williams WG, Freedom RM. Systemic venous collateral development after the bidirectional cavopulmonary anastomosis. Prevalence and predictors. J Am Coll Cardiol. 1998;32(2):502-8.

5 Kim SJ, Bae EJ, Lee JY, Lim HG, Lee C, Lee CH. Inclusion of hepatic venous drainage in patients with pulmonary arteriovenous fistulas. Ann Thorac Surg. 2009;87(2):548-53.

6 Gewillig M. The Fontan circulation. Heart. 2005;91(6):839-46.

7 Mavroudis C, Deal BJ, Backer CL, Stewart RD, Franklin WH, Tsao S, et al. J. Maxwell Chamberlain Memorial Paper for congenital heart surgery. 111 Fontan conversions with arrhythmia surgery: surgical lessons and outcomes. Ann Thorac Surg. 2007;84(5):1457-65. 